\title{
Australian Journal of \\ Chemical composition and seasonal variation of the essential oils of leaves of Garcinia gardneriana (Planchon \& Triana) Zappi (Clusiaceae)
}

\author{
Carla Maria Mariano Fernandez ${ }^{1 *}$, Fabiana Brusco Lorenzetti ${ }^{1}$, Camila Cristina Iwanaga ${ }^{1}$, Karine Zanoli \\ Bernuci ${ }^{2}$, Ludmila Pini Simões ${ }^{1}$, João Paulo Pinguello de Andrade ${ }^{3}$, Wanessa de Campos Bortolucci ${ }^{4}$, José \\ Eduardo Gonçalves ${ }^{5,6}$, Diógenes Aparício Garcia Cortez ${ }^{6}+$, Zilda Cristiani Gazim ${ }^{4}$, Benedito Prado Dias \\ Filho $^{1}$
}

\author{
${ }^{1}$ Postgraduate Program in Pharmaceutical Sciences, State University of Maringá (UEM), Maringá, Brazil \\ ${ }^{2}$ Unicesumar, Maringá, Brazil \\ ${ }^{3}$ Postgraduate Program in Chemical Engineering, Western Parana State University-(UNIOESTE), Toledo, Brazil \\ ${ }^{4}$ Postgraduate Program in Biotechnology Applied to the Agriculture, Paranaense University, Umuarama, Brazil \\ ${ }^{5}$ Postgraduate Programs in Clean Technologies, Cesumar University, Maringá, Brazil \\ ${ }^{6}$ Cesumar Institute of Science, Technology and Innovation - ICETI, Maringá, Brazil. +Deceased
}

*Corresponding author: carla.mfernandez@hotmail.com

\begin{abstract}
The seasonal factors have influence upon the secondary metabolism of plants in adaptive response to the environmental variation, leading to the biosynthesis of different compounds. Thus, the aim of the present paper was to characterize the chemical composition of the essential oil (EO) from leaves of Garcinia gardneriana (Clusiaceae), in function of the seasonality, in the period throughout the year (from January to December of 2016). The EO was obtained from fresh leaves by hydrodistillation in a modified Clevenger apparatus and analyzed using a gas chromatograph coupled to a mass spectrometer. Thereafter, the chemical constituents of the EO were evaluated by principal component analysis (PCA) and hierarchical cluster analysis (HCA). The EO yield varied differently throughout the year with the highest yield in January $(0.38 \%)$ and the lowest one in August $(0.19 \%)$. The EO composition was sesquiterpene hydrocarbons (84.78 - 99.07\%) and oxygenated sesquiterpenes $(0.45-13.80 \%)$. The major compounds found were $\alpha$-cedrene, for the months of January to November, and $\alpha$-trans-bergamotene for December. PCA and HCA analyses showed that the development stage of the plant may have altered the chemical composition of the EO, since in the flowering period the compounds $\alpha$-trans-bergamotene and $\gamma$-muurolene were higher, which are responsible for attracting pollinators and for the plant's defense. Moreover, in the fructification period, the production of compounds of the oxygenated sesquiterpenes class, namely (Z)-caryophyllene, spathulenol and caryophyllene oxide increased, what is probably related to the protection of the fruit. Thus, the EO of "bacupari" leaves had high chemical variability, which is likely related to the developmental cycle of the plant.
\end{abstract}

Keywords: "bacupari”; essential oil; seasonal variability; $\alpha$-cedrene; $\alpha$-trans-bergamotene, principal component analysis.

Introduction

Garcinia gardneriana (Planchon \& Triana) Zappi is a native tree of the Brazilian Atlantic Forest, which belongs to the Clusiaceae family, with distribution in forest formations of the Atlantic slope, from the Amazon region to Rio Grande do Sul, mainly in the rainforest (Corrêa, 1984; Lorenzi, 2002; Guimarães et al., 2004). Popularly known as "bacupari", "bacopari", "mangostão-amarelo" and "bacupari-mirim", is consumed by inhabitants of the Alto Paraná River floodplain (Porto Rico, Paraná state, Brazil) in the form of sweets, juices or in natura (Asinelli et al., 2011). In addition, it is commonly employed in the folk medicine for the treatment of inflammation, especially to skin diseases and urinary tract infections (Guimarães et al., 2004).

The bacupari is a medium-sized tree up to 10 meters high and its flowering occurs in the months between August and January. It produces yellow fruits with a white edible mucilaginous pulp, with maturing between November and February. These fruits serve as food to the Brown Capuchin
Monkey and rodents like the "cutia" (Corrêa, 1984; Guimarães et al., 2004; Asinelli et al., 2011).

Phytochemical studies with the roots and leaves of this plant showed the presence of xanthones (8-deoxygartanin, 7prenyljacareubin, rheediaxanthone-A) and bioflavonoids (volkensiflavone, fukugetin, biflavanone GB2a, biflavanone GBla, fukugeside, I3-naringenin-II8-4'-OMe-eriodictyol) (Botta et al., 1984; Delle Monache et al., 1984; Luzzi et al., 1997; Cechinel Filho et al., 2000). In relation to the fruits, the presence of benzophenones (7-epiclusianone), sesquiterpenes ( $\alpha$-copaene, $\alpha$-muurolene, $\gamma$-cadinene, cadinene), triterpenes (oleanolic acid), steroids (sitosterol, stigmasterol), methyl esters of palmitic, stearic, oleic, linoleic and linolenic acids, and sugars (galactose, glucose, fructose) was evidenced (Santos et al., 1999). However, no assessments on the chemical composition of the $G$. gardneriana essential oil (EO) have been reported in the literature. 
Secondary metabolites represent a chemical interface between plants and the environment. Thus, the chemical composition of the EO extracted from the same species and from the same part of a plant may vary significantly, since it is determined by the genetic, seasonal (different seasons of the year, mainly related to the climatic and environmental factors) and edaphic effects (Gobbo-Neto and Lopes, 2007; Morais, 2009).

Several interactions may influence in an isolated or joint way in the metabolic pathways, leading to the biosynthesis of different compounds of the essential oils. Examples of these interactions are: plant/microorganisms, plant/insect and plant/plant, factors inherent of the plant itself, such as development cycle, age, macro and micronutrients of soil, altitude, longitude, air pollution and abiotic factors such as light, temperature, rainfall index, relative humidity, and water availability (Morais, 2009; Fernandes et al., 2017).

In this way, the understanding of these factors and their relationships favor the obtainment of essential oils of better quality and more constant chemical composition, generating reliable results of biological activities (Gobbo-Neto and Lopes, 2007; Morais, 2009). Thus, the objective of this research was to characterize the chemical composition of the essential oil (EO) of leaves of G. gardneriana, as well as its seasonal variability from January 2016 to December 2016.

\section{Results and discussion}

Meteorological data of temperature, rainfall index and relative humidity of the "bacupari" leaves collection period for EO extraction, which ranged from January to December 2016, are described in Table 1 . The EO yield of bacupari leaves varied throughout the year (Table 2), with the highest yield in January $(0.38 \%)$ and the lowest one in August (0.19\%). In January, the temperatures were higher (31.59 ㅇ) compared to the other months, which may have influenced the increase of the EO yield. Morais (2009) described that temperature changes alter the production of secondary metabolites in plants and that the yield of essential oils usually increases at high temperatures.

The Pearson's correlation analysis was performed to verify the possible association between the essential oil components selected and climatic variables (temperature and rainfall). The analysis results showed that there was no significant linear relationship between the variables $(r=0.04$ and $r=0.38$, respectively). However, other factors may have an effect on the chemical composition of essential oils such as the plant origin, collection period, light, climate, vegetative stage and nutrition (Heinzmann et al., 2017).

The chemical composition of the G. gardneriana leaves EO, obtained by GC-MS, is shown in Table 2. The EO presented in its composition the sesquiterpene hydrocarbons (84.78 99.07\%) and oxygenated sesquiterpenes (0.45 - 13.80\%). Principal component analysis (PCA) and hierarchical cluster analysis (HCA) were carried out including the major EO compounds and those that presented significant quantitative variation over the months (Figures 1 and 2, Table 3). In the Figure 1, HCA is presented considering the existence of four groups, which are: cluster I: EOs from leaves collected in the months of January and June; cluster II: EOs from leaves collected in the months of February, May, August and September; cluster III: EOs from leaves collected in the months of March, April, July, October and November; and cluster IV: EO from leaves collected in the month of December. In the Figure 2, are presented only the compounds that exhibited correlation matrix above 0.8 in the PCA (Table 3).

The cluster I (January and June) and the cluster III (March, April, July, October and November), grouped the months corresponding to the vegetative period and were characterized by $\alpha$-cedrene (the major compound of EO from January to November) and the compounds $\alpha$ chamigrene, $\gamma$-elemene, $\delta$-amorphene and germacrene $B$, as shown in the PCA in Factor 1 in the Figure 2. The presence of these sesquiterpene hydrocarbons in the "bacupari" EO during the vegetative period may indicate the involvement in the defense response of the plant. According to Olayemi (2017), this class of terpenes can provide simultaneous protection against numerous predators, parasites and competitors and can act synergistically against a wide range of pathogenic fungi and bacteria.

In addition, the Cluster II grouped the months corresponding to the vegetative and flowering periods together (February, May, August and September), and was characterized by $\alpha$ trans-bergamotene and $\gamma$-muurolene in Factor 1 in the PCA (Figure 2). During the months of August and September, occurred the period of flowering, wherein concentration increases for $\alpha$-trans-bergamotene $(8.53$; $4.08 \%$, respectively), $\gamma$-muurolene $(8.80 ; 8.60 \%$, respectively) and $\gamma$ cadinene $(4.18 ; 3.70 \%$, respectively) were observed. This concentration increase at the flowering period may be related to the process of pollination and protection against microorganisms, diseases and attacks by herbivores (Cheng et al., 2007; Zhou et al., 2017).

Moreover, the cluster IV corresponded to the fruiting period, which occurred in December, and was characterized by (Z)caryophyllene, spathulenol and caryophyllene oxide. Since the concentrations of these compounds increased in this period (Table 2), it can be said that they exhibited a positive correlation in Factor 2 in the PCA analysis. The presence of these compounds in this period can be explained by their antimicrobial properties, related to the response to insect attack, mechanical damage or infection by fungal and bacterial pathogens in the fruit protection (Ulubelen et al., 1994; Matasyoh et al., 2007). On the other hand, bicyclogermacrene showed a negative correlation, decreasing its concentration in this period (Table 2). As reported by Silva et al. (2013), the increase of the concentration of spathulenol and the reduction of the amounts of bicyclogermacrene can be partially explained by biochemical factors of the enzymatic oxidation of bicyclogermacrene for the formation of spathulenol.

Furthermore, in adaptive response to possible environmental stimuli, the plant metabolism can be altered, leading to the biosynthesis of different compounds. The case of water stress condition, for instance, can induce the production of some terpenes, while the excess of water, caused by intense rainfall, may lead to loss of water-soluble substances from leaves and flowers (Gobbo-Neto and Lopes, 2007; Alves et al., 2018). In high-temperature environments, the yield of essential oils usually increases. On other hand, very high temperatures may result in excessive loss of chemical constituents by volatilization (Morais, 2009). Besides that, at higher levels of solar radiation, higher production of EO compounds may occur, since the biosynthetic reactions and the development of some structures (glandular trichomes), responsible for the production and storage, are light dependent (Gobbo-Neto and Lopes, 2007). The plant's development stage is other important factor, because in younger tissues, due to the great biosynthetic activity, the production of the essential 
Table 1. Metereological data of the period of collection of plant material of Garcinia gardneriana.

\begin{tabular}{lllll}
\hline Date & \multicolumn{2}{l}{ Average temperature $\left({ }^{\circ} \mathrm{C}\right)$} & Rainfall precipitation $(\mathrm{mm})$ & Relative humidity $(\%)$ \\
\cline { 2 - 3 } & Minimum & Maximum & & \\
\hline $01 / 31 / 2016$ & $21.93 \pm 1.30$ & $31.59 \pm 2.27$ & 236.3 & $72.82 \pm 17.09$ \\
$02 / 29 / 2016$ & $22.48 \pm 1.37$ & $31.23 \pm 3.33$ & 260.9 & $80.91 \pm 9.55$ \\
$03 / 31 / 2016$ & $20.01 \pm 2.52$ & $30.20 \pm 2.92$ & 114.3 & $69.09 \pm 11.25$ \\
$04 / 30 / 2016$ & $20.89 \pm 5.90$ & $31.64 \pm 5.67$ & 78.0 & $58.66 \pm 12.13$ \\
$05 / 31 / 2016$ & $14.88 \pm 3.09$ & $22.86 \pm 3.53$ & 332.1 & $80.42 \pm 17.94$ \\
$06 / 30 / 2016$ & $12.53 \pm 3.82$ & $21.54 \pm 3.90$ & 51.9 & $70.23 \pm 19.60$ \\
$07 / 31 / 2016$ & $14.40 \pm 4.50$ & $25.86 \pm 4.27$ & 61.2 & $58.13 \pm 11.58$ \\
$08 / 31 / 2016$ & $15.48 \pm 3.55$ & $26.08 \pm 5.15$ & 164.3 & $64.18 \pm 20.84$ \\
$09 / 30 / 2016$ & $15.76 \pm 3.81$ & $27.29 \pm 5.00$ & 38.4 & $54.54 \pm 16.40$ \\
$10 / 31 / 2016$ & $17.99 \pm 3.79$ & $29.09 \pm 4.11$ & 362.8 & $65.17 \pm 16.13$ \\
$11 / 30 / 2016$ & $19.76 \pm 2.68$ & $31.11 \pm 2.66$ & 77.5 & $60.55 \pm 13.48$ \\
$12 / 31 / 2016$ & $20.15 \pm 1.62$ & $30.30 \pm 3.08$ & 167.2 & $81.79 \pm 11.03$ \\
\hline
\end{tabular}

Average \pm standard deviation.

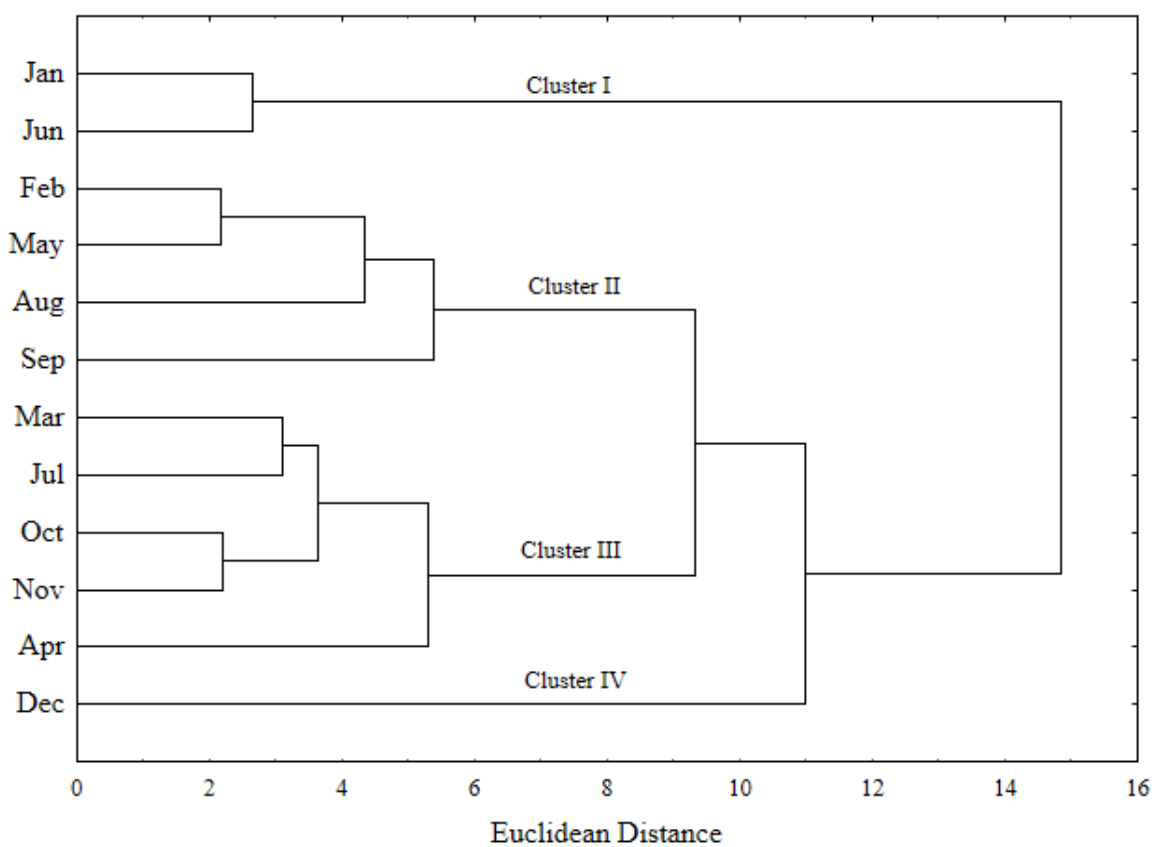

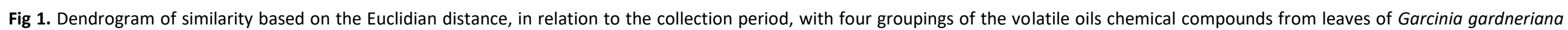

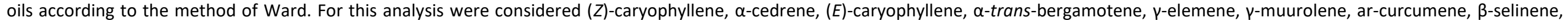
germacrene $D$, bicyclogermacrene, $\alpha$-chamigrene, $\delta$-amorphene, $\gamma$-cadinene, $\beta$-curcumene, $(Z)-\gamma$-bisabolene, $\delta$-cadinene, germacrene $B$, spathulenol and caryophyllene oxide. 
Table 2. Chemical constituents of the essential oils of leaves and fruits of Garcinia gardneriana.

\begin{tabular}{|c|c|c|c|c|c|c|c|c|c|c|c|c|c|c|c|}
\hline \multirow[b]{2}{*}{ Peak } & \multirow[b]{2}{*}{${ }^{A}$ Compound } & \multirow[b]{2}{*}{$\begin{array}{l}{ }^{\mathrm{a}} \mathrm{RI} \\
\text { Literature }\end{array}$} & \multirow[b]{2}{*}{$\begin{array}{l}{ }^{\mathrm{b}} \mathrm{RI} \\
\text { Calculated }\end{array}$} & \multicolumn{12}{|c|}{ Area (\%) } \\
\hline & & & & Jan & Feb & Mar & Apr & May & Jun & Jul & Aug & Sep & Oct & Nov & Dec \\
\hline 1 & $\delta$-Elemene & 1338 & 1336 & 0.36 & 0.90 & 0.61 & 0.44 & 1.28 & 0.69 & 0.58 & 0.29 & 1.47 & 0.78 & 1.05 & 0.23 \\
\hline 2 & $\alpha$-Cubebene & 1348 & 1348 & 0.56 & 0.56 & 0.32 & 0.18 & 0.36 & 0.53 & 0.36 & 0.53 & 0.45 & 0.43 & 0.28 & 0.49 \\
\hline 3 & n.i. & - & 1353 & $\mathrm{t}$ & $\mathrm{t}$ & 0.09 & $\mathrm{t}$ & 0.11 & $\mathrm{t}$ & 0.08 & $\mathrm{t}$ & $\mathrm{t}$ & 0.08 & 0.06 & $\mathrm{t}$ \\
\hline 4 & n.i. & - & 1366 & $\mathrm{t}$ & 0.18 & 0.08 & $\mathrm{t}$ & 0.11 & 0.05 & 0.12 & 0.20 & $\mathrm{t}$ & 0.08 & 0.14 & 0.13 \\
\hline 5 & $\alpha$-Ylangene & 1375 & 1371 & 0.21 & 0.34 & 0.24 & 0.12 & 0.26 & 0.21 & 0.23 & 0.39 & 0.29 & 0.26 & 0.20 & 0.32 \\
\hline 6 & $\alpha$-Copaene & 1376 & 1375 & 0.89 & 1.38 & 0.83 & 0.51 & 1.09 & 0.88 & 0.79 & 1.37 & 1.48 & 1.10 & 0.83 & 1.39 \\
\hline 7 & Daucene & 1381 & 1377 & $\mathrm{t}$ & $\mathrm{t}$ & $\mathrm{t}$ & $\mathrm{t}$ & $\mathrm{t}$ & $\mathrm{t}$ & $\mathrm{t}$ & $\mathrm{t}$ & 0.21 & 0.22 & 0.23 & $t$ \\
\hline 8 & $\beta$-Cubebene & 1388 & 1379 & 0.34 & 0.19 & 0.26 & 0.21 & 0.19 & 0.31 & 0.26 & 0.21 & 0.12 & $\mathrm{t}$ & $\mathrm{t}$ & 0.24 \\
\hline 9 & $(-)-\beta$-Bourbonene & 1388 & 1382 & 0.20 & 0.54 & 0.31 & 0.16 & 0.42 & 0.11 & 0.41 & 0.56 & 0.93 & 1.03 & 0.49 & 0.89 \\
\hline 10 & n.i. & - & 1385 & $\mathrm{t}$ & 0.28 & 0.14 & 0.07 & 0.23 & $\mathrm{t}$ & $\mathrm{t}$ & 0.15 & 0.29 & $\mathrm{t}$ & 0.29 & 0.22 \\
\hline 11 & $\beta$-Elemene & 1390 & 1393 & 1.03 & 3.63 & 1.83 & 1.71 & 3.08 & 0.02 & 0.16 & 2.07 & 3.52 & 2.37 & 3.94 & 2.77 \\
\hline 12 & 7-epi-Sesquithujene & 1391 & 1394 & 0.11 & 0.11 & $\mathrm{t}$ & 0.86 & $\mathrm{t}$ & 1.39 & 2.11 & 0.10 & 0.56 & 0.09 & 0.09 & 0.10 \\
\hline 13 & n.i. & & 1402 & $\mathrm{t}$ & $\mathrm{t}$ & $\mathrm{t}$ & $\mathrm{t}$ & $\mathrm{t}$ & 0.11 & 0.09 & $\mathrm{t}$ & $\mathrm{t}$ & $\mathrm{t}$ & $\mathrm{t}$ & $\mathrm{t}$ \\
\hline 14 & (Z)-Caryophyllene & 1408 & 1406 & - & - & - & - & - & - & - & - & - & - & - & 7.20 \\
\hline 15 & $\alpha$-Cedrene & 1411 & 1417 & 40.29 & 9.67 & 18.94 & 25.13 & 7.70 & 30.48 & 20.87 & 13.32 & 13.45 & 15.85 & 21.32 & 6.22 \\
\hline 16 & $\alpha$-cis-Bergamotene & 1412 & 1418 & 2.01 & 2.41 & 2.81 & 1.44 & 2.64 & 2.09 & 2.93 & 2.60 & 1.60 & 2.62 & 2.47 & 3.48 \\
\hline 17 & n.i. & - & 1420 & 0.32 & $\mathrm{t}$ & 0.23 & 0.59 & $\mathrm{t}$ & 0.32 & 0.23 & $\mathrm{t}$ & $\mathrm{t}$ & 0.18 & 0.20 & $\mathrm{t}$ \\
\hline 18 & (E)-Caryophyllene & 1419 & 1421 & $\mathrm{t}$ & 7.17 & 5.86 & 13.22 & 6.61 & 4.09 & 6.19 & 7.32 & 7.40 & 5.92 & 6.52 & 6.41 \\
\hline 19 & $\beta$-Copaene & 1432 & 1431 & 0.49 & 1.26 & 0.78 & 0.41 & $\mathrm{t}$ & 0.52 & 0.85 & 1.18 & 1.39 & 1.03 & 0.81 & 1.07 \\
\hline 20 & $\beta$-Gurjunene & 1433 & 1434 & 0.86 & 0.17 & 0.54 & 4.07 & 0.95 & 0.89 & 0.42 & 0.35 & 0.20 & 0.28 & 0.36 & 0.16 \\
\hline 21 & $\alpha$-trans-Bergamotene & 1434 & 1435 & $\mathrm{t}$ & 7.26 & 5.99 & 4.32 & 8.46 & $\mathrm{t}$ & 6.00 & 8.53 & 4.08 & 5.97 & 4.71 & 9.51 \\
\hline 22 & $\gamma$-Elemene & 1436 & 1436 & 7.94 & 0.80 & 2.83 & $\mathrm{t}$ & 0.63 & 6.35 & 3.41 & 1.11 & 2.22 & 2.48 & 3.30 & 0.64 \\
\hline 23 & $\alpha$-Guaiene & 1439 & 1438 & 0.21 & 0.28 & 0.20 & $\mathrm{t}$ & 0.25 & 1.45 & 0.22 & 0.31 & 0.24 & 0.23 & 0.19 & 0.21 \\
\hline 24 & Aromadendrene & 1441 & 1443 & 0.21 & $\mathrm{t}$ & 0.27 & $\mathrm{t}$ & 0.34 & 0.27 & $\mathrm{t}$ & 0.38 & 0.17 & 0.51 & 0.22 & 0.24 \\
\hline 25 & (Z)- $\beta$-Farnesene & 1442 & 1446 & $\mathrm{t}$ & 0.24 & $\mathrm{t}$ & $\mathrm{t}$ & $\mathrm{t}$ & $t$ & $\mathrm{t}$ & $\mathrm{t}$ & $\mathrm{t}$ & $\mathrm{t}$ & $\mathrm{t}$ & $\mathrm{t}$ \\
\hline 26 & $\alpha$-neo-Clovene & 1452 & 1448 & $\mathrm{t}$ & 0.24 & 0.56 & $\mathrm{t}$ & 0.36 & 0.28 & 0.47 & $t$ & 0.17 & $\mathrm{t}$ & 0.22 & 0.39 \\
\hline 27 & $\alpha$-Humulene & 1454 & 1452 & 1.10 & 2.65 & 2.11 & 2.04 & 2.70 & 1.30 & 2.42 & 2.32 & 2.68 & 1.92 & 2.58 & 2.47 \\
\hline 28 & n.i. & - & 1458 & $\mathrm{t}$ & $\mathrm{t}$ & $\mathrm{t}$ & $\mathrm{t}$ & $\mathrm{t}$ & 0.24 & $\mathrm{t}$ & 0.41 & 0.38 & 0.38 & 0.10 & 0.52 \\
\hline 29 & $(E)-\beta$-Farnesene & 1456 & 1462 & $\mathrm{t}$ & $\mathrm{t}$ & 1.39 & 0.14 & 1.83 & 0.61 & 1.29 & 1.29 & 1.02 & 1.24 & 1.22 & 1.32 \\
\hline 30 & allo-Aromadendrene & 1460 & 1463 & 0.23 & 0.43 & 0.40 & $\mathrm{t}$ & 0.47 & $\mathrm{t}$ & 0.41 & $\mathrm{t}$ & $\mathrm{t}$ & $\mathrm{t}$ & 0.35 & $\mathrm{t}$ \\
\hline 31 & $\alpha$-Acoradiene & 1466 & 1464 & 0.54 & 1.47 & 0.51 & 0.39 & $\mathrm{t}$ & 0.60 & 0.43 & 0.27 & 0.44 & 0.43 & 0.41 & 0.34 \\
\hline 32 & $\beta$-Acoradiene & 1470 & 1471 & 0.63 & 0.28 & 0.58 & 0.28 & $\mathrm{t}$ & 0.90 & 0.55 & 0.34 & 0.36 & 0.45 & 0.48 & 0.31 \\
\hline 33 & $\gamma$-Muurolene & 1479 & 1479 & 3.34 & 8.60 & 4.64 & 3.30 & 6.63 & 3.59 & 4.56 & 8.80 & 8.60 & 4.55 & 5.27 & 6.96 \\
\hline 34 & ar-Curcumene & 1480 & 1480 & $\mathrm{t}$ & 4.64 & 3.63 & 0.79 & 3.45 & $\mathrm{t}$ & $\mathrm{t}$ & 9.34 & 0.20 & 2.32 & 1.13 & 7.07 \\
\hline 35 & Amorpha-4,7(11)- diene & 1481 & 1481 & 0.37 & 4.70 & 3.52 & 2.64 & 6.45 & 0.81 & 2.65 & 1.15 & 2.60 & 1.15 & 1.19 & 1.21 \\
\hline 36 & $\gamma$-Curcumene & 1482 & 1482 & 0.22 & $\mathrm{t}$ & $\mathrm{t}$ & $\mathrm{t}$ & $\mathrm{t}$ & 1.63 & $\mathrm{t}$ & $\mathrm{t}$ & $\mathrm{t}$ & $\mathrm{t}$ & $\mathrm{t}$ & $\mathrm{t}$ \\
\hline 37 & Germacrene D & 1485 & 1485 & $\mathrm{t}$ & $\mathrm{t}$ & $\mathrm{t}$ & $\mathrm{t}$ & 0.31 & $t$ & $\mathrm{t}$ & $\mathrm{t}$ & $\mathrm{t}$ & 5.58 & 3.84 & 3.74 \\
\hline 38 & $\beta$-Selinene & 1490 & 1490 & 4.16 & $\mathrm{t}$ & $\mathrm{t}$ & 2.71 & $\mathrm{t}$ & 3.16 & 4.09 & $\mathrm{t}$ & 7.07 & $\mathrm{t}$ & $\mathrm{t}$ & 3.34 \\
\hline 39 & $\delta$-Selinene & 1492 & 1491 & $\mathrm{t}$ & 0.39 & $\mathrm{t}$ & $\mathrm{t}$ & $\mathrm{t}$ & 0.42 & $\mathrm{t}$ & $\mathrm{t}$ & $\mathrm{t}$ & $\mathrm{t}$ & $\mathrm{t}$ & $\mathrm{t}$ \\
\hline 40 & $\alpha$-Zingiberene & 1493 & 1494 & 0.90 & 2.27 & 2.55 & 1.23 & 3.35 & 1.27 & 2.43 & 0.68 & 1.38 & 2.08 & 1.69 & $\mathrm{t}$ \\
\hline 41 & Bicyclogermacrene & 1498 & 1496 & 4.63 & 8.06 & 5.41 & 4.72 & 7.57 & 5.71 & 5.54 & 6.95 & 7.78 & 5.26 & 5.78 & $\mathrm{t}$ \\
\hline 42 & $\alpha$-Muurolene & 1500 & 1501 & 0.31 & 0.71 & 0.47 & $\mathrm{t}$ & 0.67 & 0.42 & $\mathrm{t}$ & 0.72 & 0.60 & 0.55 & 0.26 & 0.78 \\
\hline 43 & $\beta$-Himachalene & 1500 & 1508 & 0.25 & 0.55 & 0.50 & 0.27 & 0.65 & $\mathrm{t}$ & 0.40 & 0.21 & 0.44 & 0.46 & 0.40 & $\mathrm{t}$ \\
\hline 44 & $\alpha$-Chamigrene & 1503 & 1509 & 12.32 & 6.04 & 7.44 & 13.40 & 5.02 & 11.67 & 10.48 & 6.44 & 6.44 & 9.61 & 9.47 & 7.84 \\
\hline 45 & $\beta$-Bisabolene & 1505 & 1509 & 0.20 & $\mathrm{t}$ & $\mathrm{t}$ & $\mathrm{t}$ & $\mathrm{t}$ & 0.67 & $\mathrm{t}$ & $\mathrm{t}$ & $\mathrm{t}$ & $\mathrm{t}$ & $\mathrm{t}$ & $t$ \\
\hline 46 & $\delta$-Amorphene & 1512 & 1509 & 2.20 & $\mathrm{t}$ & $\mathrm{t}$ & $\mathrm{t}$ & $\mathrm{t}$ & 2.56 & $\mathrm{t}$ & $\mathrm{t}$ & $\mathrm{t}$ & $\mathrm{t}$ & $\mathrm{t}$ & $\mathrm{t}$ \\
\hline 47 & n.i. & - & 1512 & 0.26 & $t$ & $\mathrm{t}$ & $\mathrm{t}$ & $\mathrm{t}$ & $t$ & $\mathrm{t}$ & $\mathrm{t}$ & $\mathrm{t}$ & $\mathrm{t}$ & $\mathrm{t}$ & $\mathrm{t}$ \\
\hline 48 & -Cadinene & 1513 & 1513 & 1.82 & 2.95 & 2.98 & $\mathrm{t}$ & 3.21 & $\mathrm{t}$ & $\mathrm{t}$ & 4.18 & 3.70 & 2.49 & $\mathrm{t}$ & 2.51 \\
\hline
\end{tabular}




\begin{tabular}{|c|c|c|c|c|c|c|c|c|c|c|c|c|c|c|c|}
\hline 49 & $\beta$-Curcumene & 1515 & 1517 & $\mathrm{t}$ & 3.10 & 2.97 & 3.31 & 6.23 & $\mathrm{t}$ & 2.12 & $\mathrm{t}$ & $\mathrm{t}$ & 2.63 & 2.31 & $\mathrm{t}$ \\
\hline 50 & (Z)- $\psi$-Bisabolene & 1515 & 1519 & $\mathrm{t}$ & 4.39 & 4.26 & 3.64 & 5.53 & $\mathrm{t}$ & 3.67 & 2.18 & 2.90 & 4.05 & 3.37 & $\mathrm{t}$ \\
\hline 51 & $\delta$-Cadinene & 1523 & 1526 & $\mathrm{t}$ & 6.39 & 4.38 & 1.07 & 5.50 & 2.22 & 4.11 & 6.45 & 5.16 & 4.51 & 3.32 & 2.44 \\
\hline 52 & trans-Cadina-1,4-diene & 1534 & 1533 & $\mathrm{t}$ & 0.96 & 1.28 & t & 0.97 & $\mathrm{t}$ & 0.95 & 1.02 & 0.69 & 0.87 & 0.71 & 0.39 \\
\hline 53 & $\alpha$-Cadinene & 1538 & 1537 & $\mathrm{t}$ & 0.72 & 0.62 & 0.13 & 0.57 & 0.55 & 0.53 & 0.86 & 0.58 & 0.55 & 0.40 & 0.56 \\
\hline 54 & $\alpha$-Calacorene & 1545 & 1540 & $\mathrm{t}$ & 0.12 & 0.09 & $\mathrm{t}$ & 0.05 & $\mathrm{t}$ & 0.03 & $\mathrm{t}$ & $\mathrm{t}$ & 0.17 & $\mathrm{t}$ & $\mathrm{t}$ \\
\hline 55 & Selina-3,7(11)-diene & 1546 & 1541 & 2.13 & 0.19 & 0.67 & 0.76 & 0.09 & 2.00 & 0.42 & 0.67 & 0.15 & 0.25 & 0.28 & 0.19 \\
\hline 56 & n.i. & - & 1545 & 0.13 & $\mathrm{t}$ & $\mathrm{t}$ & $\mathrm{t}$ & $\mathrm{t}$ & $\mathrm{t}$ & 0.14 & 0.12 & $\mathrm{t}$ & $\mathrm{t}$ & $\mathrm{t}$ & 0.37 \\
\hline 57 & n.i. & - & 1546 & $\mathrm{t}$ & $\mathrm{t}$ & 0.16 & $\mathrm{t}$ & $\mathrm{t}$ & $\mathrm{t}$ & $\mathrm{t}$ & $\mathrm{t}$ & $\mathrm{t}$ & $\mathrm{t}$ & $\mathrm{t}$ & $\mathrm{t}$ \\
\hline 58 & Elemol & 1549 & 1547 & $\mathrm{t}$ & 0.14 & $\mathrm{t}$ & $\mathrm{t}$ & 0.23 & $\mathrm{t}$ & $\mathrm{t}$ & $\mathrm{t}$ & 0.10 & 0.18 & 0.15 & $\mathrm{t}$ \\
\hline 59 & Germacrene B & 1561 & 1554 & 7.19 & 1.00 & 3.65 & 5.30 & 0.93 & 8.40 & 3.77 & 1.32 & 2.57 & 2.87 & 3.73 & 0.73 \\
\hline 60 & n.i. & - & 1561 & $\mathrm{t}$ & $\mathrm{t}$ & 0.06 & $\mathrm{t}$ & 0.10 & 0.21 & $\mathrm{t}$ & $\mathrm{t}$ & t & $\mathrm{t}$ & $\mathrm{t}$ & $\mathrm{t}$ \\
\hline 61 & $\beta$-Calacorene & 1565 & 1562 & $\mathrm{t}$ & $\mathrm{t}$ & $\mathrm{t}$ & $\mathrm{t}$ & $\mathrm{t}$ & $\mathrm{t}$ & $\mathrm{t}$ & $\mathrm{t}$ & $\mathrm{t}$ & 0.16 & $\mathrm{t}$ & $\mathrm{t}$ \\
\hline 62 & Maaliol & 1567 & 1564 & $\mathrm{t}$ & $\mathrm{t}$ & $\mathrm{t}$ & $\mathrm{t}$ & $\mathrm{t}$ & $\mathrm{t}$ & $\mathrm{t}$ & $\mathrm{t}$ & $\mathrm{t}$ & $\mathrm{t}$ & 0.14 & 0.20 \\
\hline 63 & Spathulenol & 1578 & 1573 & 0.42 & 0.16 & 0.21 & $\mathrm{t}$ & 0.35 & $\mathrm{t}$ & 0.27 & 1.07 & 0.32 & 0.36 & 0.19 & 2.70 \\
\hline 64 & Caryophyllene oxide & 1583 & 1577 & $\mathrm{t}$ & 0.48 & 0.54 & 0.27 & 0.37 & $\mathrm{t}$ & 0.52 & 1.09 & 0.43 & 0.74 & 0.53 & 4.46 \\
\hline 65 & n.i. & - & 1579 & $\mathrm{t}$ & $\mathrm{t}$ & t & t & $\mathrm{t}$ & $\mathrm{t}$ & $\mathrm{t}$ & $\mathrm{t}$ & 0.34 & $\mathrm{t}$ & $\mathrm{t}$ & $\mathrm{t}$ \\
\hline 66 & Globulol & 1590 & 1581 & 0.22 & 0.13 & 0.20 & $\mathrm{t}$ & 0.39 & $\mathrm{t}$ & 0.11 & 0.13 & 0.25 & 0.15 & 0.24 & 0.31 \\
\hline 67 & $\beta$-Copaen-4- $\alpha$-ol & 1590 & 1588 & $\mathrm{t}$ & 0.12 & 0.27 & 0.18 & 0.39 & $\mathrm{t}$ & t & $\mathrm{t}$ & 0.28 & 0.42 & 0.39 & 0.47 \\
\hline 68 & n.i. & - & 1589 & 0.11 & t & 0.27 & $\mathrm{t}$ & $\mathrm{t}$ & $\mathrm{t}$ & 0.09 & $\mathrm{t}$ & 0.17 & $\mathrm{t}$ & $\mathrm{t}$ & t \\
\hline 69 & Cubeban-11-ol & 1595 & 1591 & $\mathrm{t}$ & $\mathrm{t}$ & t & $\mathrm{t}$ & $\mathrm{t}$ & $\mathrm{t}$ & $\mathrm{t}$ & $\mathrm{t}$ & $\mathrm{t}$ & 0.13 & 0.13 & 0.22 \\
\hline 70 & Rosifoliol & 1600 & 1599 & $\mathrm{t}$ & 0.06 & $\mathrm{t}$ & $\mathrm{t}$ & $\mathrm{t}$ & $\mathrm{t}$ & 0.11 & $\mathrm{t}$ & 0.13 & 0.10 & 0.14 & 0.21 \\
\hline 71 & n.i. & - & 1600 & $\mathrm{t}$ & $\mathrm{t}$ & $\mathrm{t}$ & $\mathrm{t}$ & $\mathrm{t}$ & $\mathrm{t}$ & t & t & $\mathrm{t}$ & 0.05 & $\mathrm{t}$ & 0.63 \\
\hline 72 & $\beta$-Oplopenone & 1607 & 1605 & 0.16 & 0.11 & 0.18 & $\mathrm{t}$ & 0.22 & $\mathrm{t}$ & 0.13 & 0.16 & 0.28 & 0.33 & 0.38 & 0.66 \\
\hline 73 & Junenol & 1619 & 1610 & t & t & t & $\mathrm{t}$ & $\mathrm{t}$ & $\mathrm{t}$ & $\mathrm{t}$ & $\mathrm{t}$ & 0.18 & $\mathrm{t}$ & 0.12 & 0.59 \\
\hline 74 & 1-epi-Cubenol & 1628 & 1626 & $\mathrm{t}$ & $\mathrm{t}$ & $\mathrm{t}$ & $\mathrm{t}$ & $\mathrm{t}$ & $\mathrm{t}$ & 0.26 & $\mathrm{t}$ & 0.10 & 0.20 & 0.05 & 0.63 \\
\hline 75 & allo-aromadendrene epoxide & 1641 & 1632 & $\mathrm{t}$ & $\mathrm{t}$ & $\mathrm{t}$ & $\mathrm{t}$ & $\mathrm{t}$ & $\mathrm{t}$ & 0.10 & 0.17 & 0.12 & 0.13 & 0.10 & 0.59 \\
\hline 76 & Selina-3,11-dien-6- $\alpha$-ol & 1644 & 1641 & $\mathrm{t}$ & $\mathrm{t}$ & $\mathrm{t}$ & $\mathrm{t}$ & $\mathrm{t}$ & $\mathrm{t}$ & $\mathrm{t}$ & t & t & $\mathrm{t}$ & $\mathrm{t}$ & 0.43 \\
\hline 77 & $\alpha$-Muurolol & 1646 & 1646 & $\mathrm{t}$ & $\mathrm{t}$ & 0.17 & $\mathrm{t}$ & 0.22 & $\mathrm{t}$ & 0.15 & 0.20 & 0.44 & 0.35 & 0.25 & $\mathrm{t}$ \\
\hline 78 & n.i. & - & 1647 & $\mathrm{t}$ & $\mathrm{t}$ & t & $\mathrm{t}$ & $\mathrm{t}$ & $\mathrm{t}$ & $\mathrm{t}$ & 0.10 & t & $\mathrm{t}$ & $\mathrm{t}$ & 0.19 \\
\hline 79 & $\alpha$-Cadinol & 1654 & 1654 & 0.13 & 0.19 & $\mathrm{t}$ & $\mathrm{t}$ & $\mathrm{t}$ & $\mathrm{t}$ & $\mathrm{t}$ & $\mathrm{t}$ & 0.84 & 0.54 & 0.66 & $\mathrm{t}$ \\
\hline 80 & Selin-11-en-4- $\alpha$-ol & 1659 & 1655 & $\mathrm{t}$ & 0.37 & 0.22 & $\mathrm{t}$ & 0.47 & $\mathrm{t}$ & 0.25 & 0.16 & $\mathrm{t}$ & $\mathrm{t}$ & $\mathrm{t}$ & 0.99 \\
\hline 81 & cis-Calamenen-10-ol & 1661 & 1656 & $\mathrm{t}$ & $\mathrm{t}$ & $\mathrm{t}$ & $\mathrm{t}$ & $\mathrm{t}$ & $\mathrm{t}$ & $\mathrm{t}$ & $\mathrm{t}$ & $\mathrm{t}$ & $\mathrm{t}$ & $\mathrm{t}$ & 0.24 \\
\hline 82 & trans-Calamenen-10-ol & 1669 & 1661 & $\mathrm{t}$ & $\mathrm{t}$ & $\mathrm{t}$ & $\mathrm{t}$ & $\mathrm{t}$ & $\mathrm{t}$ & $\mathrm{t}$ & $\mathrm{t}$ & $\mathrm{t}$ & $\mathrm{t}$ & $\mathrm{t}$ & 0.58 \\
\hline 83 & n.i. & - & 1665 & $\mathrm{t}$ & $\mathrm{t}$ & $\mathrm{t}$ & $\mathrm{t}$ & $\mathrm{t}$ & $\mathrm{t}$ & 0.14 & 0.21 & $\mathrm{t}$ & $\mathrm{t}$ & $\mathrm{t}$ & $\mathrm{t}$ \\
\hline 84 & Cadalene & 1676 & 1674 & $\mathrm{t}$ & $\mathrm{t}$ & $\mathrm{t}$ & $\mathrm{t}$ & $\mathrm{t}$ & $\mathrm{t}$ & $\mathrm{t}$ & $\mathrm{t}$ & $\mathrm{t}$ & 0.15 & $\mathrm{t}$ & $\mathrm{t}$ \\
\hline 85 & epi- $\alpha$-Bisabolol & 1684 & 1684 & $\mathrm{t}$ & $\mathrm{t}$ & $\mathrm{t}$ & $\mathrm{t}$ & $\mathrm{t}$ & $\mathrm{t}$ & t & $\mathrm{t}$ & $\mathrm{t}$ & $\mathrm{t}$ & 0.19 & $\mathrm{t}$ \\
\hline 86 & Eudesm-7(11)-en-4-ol & 1700 & 1698 & $\mathrm{t}$ & $\mathrm{t}$ & $\mathrm{t}$ & $\mathrm{t}$ & $\mathrm{t}$ & $\mathrm{t}$ & 0.07 & $\mathrm{t}$ & $\mathrm{t}$ & 0.09 & 0.13 & 0.51 \\
\hline 87 & n.i. & - & 1699 & $\mathrm{t}$ & $\mathrm{t}$ & $\mathrm{t}$ & $\mathrm{t}$ & $\mathrm{t}$ & $t$ & 0.05 & $\mathrm{t}$ & $\mathrm{t}$ & $\mathrm{t}$ & $\mathrm{t}$ & t \\
\hline \multicolumn{4}{|c|}{ Sesquiterpene hydrocarbons (\%) } & 98.57 & 97.50 & 97.20 & 98.90 & 96.80 & 99.07 & 97.10 & 95.82 & 95.32 & 95.51 & 95.62 & 84.78 \\
\hline \multicolumn{4}{|c|}{ Oxygenated sesquiterpenes (\%) } & 0.93 & 1.76 & 1.78 & 0.45 & 2.65 & $\mathrm{t}$ & 1.95 & 3.19 & 3.48 & 3.71 & 3.79 & 13.80 \\
\hline \multicolumn{4}{|c|}{ Total identifed (\%) } & 99.50 & 99.26 & 99.21 & 99.35 & 99.45 & 99.07 & 99.05 & 99.01 & 98.80 & 99.22 & 99.41 & 98.58 \\
\hline \multicolumn{4}{|c|}{ Yield (\%) } & 0.38 & 0.30 & 0.31 & 0.25 & 0.26 & 0.30 & 0.35 & 0.19 & 0.21 & 0.24 & 0.28 & 0.24 \\
\hline
\end{tabular}




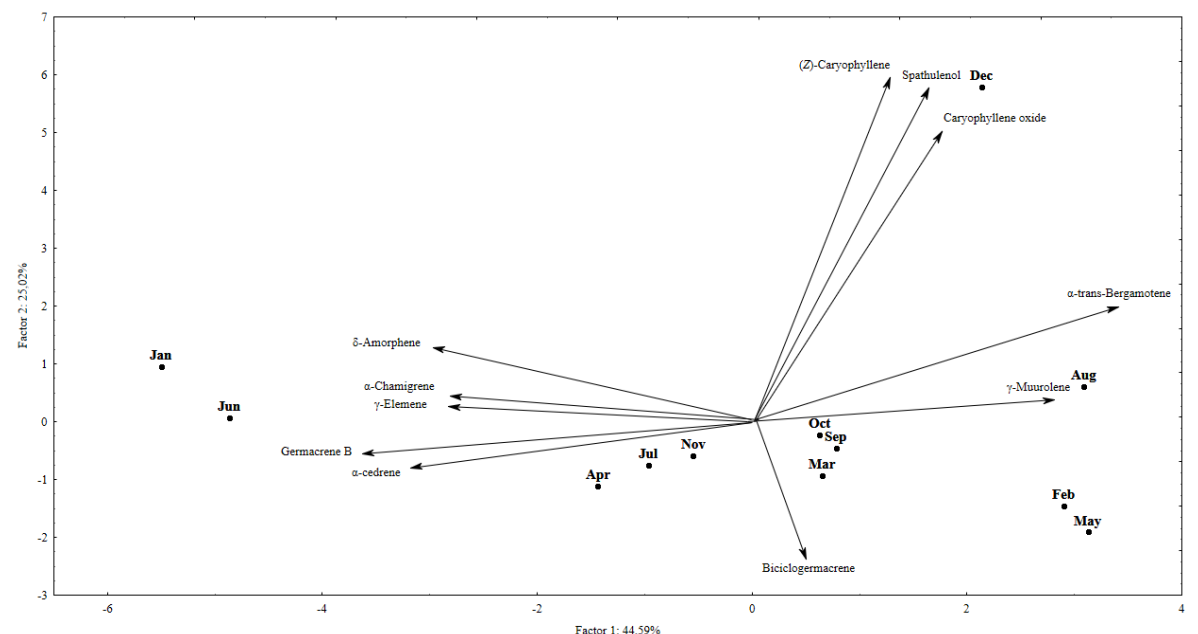

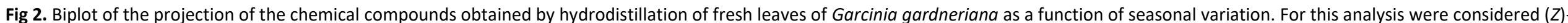

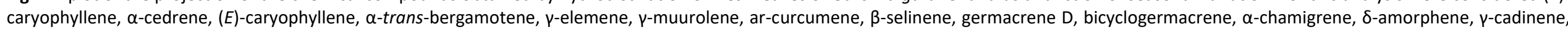
$\beta$-curcumene, $(Z)$ - $\psi$-bisabolene, $\delta$-cadinene, germacrene $B$, spathulenol and caryophyllene oxide.

Table 3. Eigenvalues, variance and correlation matrix for selected compounds of essential oil of Garcinia gardneriana.

\begin{tabular}{|c|c|c|c|c|}
\hline & Eigenvalues & Total variance (\%) & Eigenvalues accumulated & Accumulated (\%) \\
\hline Factor 1 & 8.47 & 44.59 & 8.47 & 44.59 \\
\hline Factor 2 & 4.75 & 25.02 & 13.23 & 69.61 \\
\hline \multicolumn{5}{|l|}{ Correlation matrix } \\
\hline Compound & & Factor 1 & Factor 2 & \\
\hline (Z)-Caryophyllene & & 0.32 & 0.90 & \\
\hline$\alpha$-Cedrene & & -0.96 & -0.38 & \\
\hline (E)-Caryophyllene & & 0.44 & -0.23 & \\
\hline$\alpha$-trans-Bergamotene & & 0.95 & 0.11 & \\
\hline$\gamma$-Elemene & & -0.86 & 0.09 & \\
\hline v-Muurolene & & 0.80 & 0.14 & \\
\hline ar-Curcumene & & 0.76 & 0.32 & \\
\hline$\beta$-Selinene & & -0.42 & 0.31 & \\
\hline Germacrene D & & 0.21 & 0.40 & \\
\hline Bicyclogermacrene & & 0.10 & -0.90 & \\
\hline$\alpha$-Chamigrene & & -0.84 & 0.16 & \\
\hline$\delta$-Amorphene & & -0.83 & 0.17 & \\
\hline$\gamma$-Cadinene & & 0.66 & 0.10 & \\
\hline$\beta$-Curcumene & & 0.33 & -0.62 & \\
\hline (Z)- $\gamma$-Bisabolene & & 0.50 & -0.77 & \\
\hline$\delta$-Cadinene & & 0.77 & -0.44 & \\
\hline Germacrene B & & -0.98 & -0.02 & \\
\hline spathulenol & & 0.45 & 0.88 & \\
\hline Caryophyllene oxide & & 0.50 & 0.85 & \\
\hline
\end{tabular}


oils might increase. Similarly, the nutritional state of the plant may have an influence, since the excess or deficiency of nutrients significantly affects the production of active substances (Morais, 2009). In this sense, the effects of the vegetative stage on the EO composition of some medicinal plants have been investigated. The variations associated with the plant's development period can influence the production of active substances (Faraji et al. 2016). In a previous assessment, performed by Abu-Darwish et al. (2012), it was observed that the chemical composition of the Thymus vulgaris EO varied during different growth stages. Hazzoumi et al. (2015) evaluated the Pelargonium sp EO composition during the different phases of growth. The results showed that the vegetative stage influenced upon the chemical composition of the EO, since there was a qualitative change within this period, with a balance of appearance and disappearance of some compounds along with some changes in the contents of the main compounds (menthol, isogeraniol, menthene and eremophilene). The major compounds of $G$. gardneriana leaves volatile oils were found in the literature as compounds of other species of Garcinia. The EO of fruits of Garcinia huillensis collected in Gutu and Rusape (Zimbabwe) showed as majority class the sesquiterpenes (88.5-89.2\%), and the major compounds were $\beta$-caryophyllene (12.6-53.9\%), $\alpha$-humulene (10.123.0\%) and valencene (4.0-18.2\%) (Chagonda and Chalchat, 2005). The EO of Garcinia atroviridis fruits presented sesquiterpenes as major class and (-)- $\beta$-caryophyllene (23.8\%), $\beta$-caryophyllene alcohol (15.6\%) and $\alpha$-humulene $(10.7 \%)$ as major compounds (Tan et al. 2013), whereas the EO of $G$. atroviridis leaves exhibited ( $E)$ - $\beta$-farnesene $(58.5 \%)$ and $\beta$-caryophyllene (16.9\%) as major constituents (Tan et al. 2018). In a study carried out by Aboaba et al. (2014), with the EO of Garcinia mangostana leaves and stem-bark, it was reported that the sesquiterpenes represented the major class, whereas $\beta$-caryophyllene was the most abundant constituent, which was common to both oils.

In conclusion, the results reported herein may suggest that the EO of "bacupari" leaves has high chemical variability, probably related to the developmental cycle of the plant. This conclusion was based on the fact that in the flowering period the compounds $\alpha$-trans-bergamotene, $\gamma$-muurolene and $\gamma$-cadinene were found, which may be responsible for attracting pollinators and plant's defense. In the fructification period, however, there was an increase in the production of compounds of the class of oxygenated sesquiterpenes, such as (Z)-caryophyllene, spathulenol e caryophyllene oxide, probably related to the protection of the fruit.

\section{Materials and methods}

\section{Plant materials}

Leaves of G. gardneriana were collected monthly in the period from January 2016 to December 2016 in the rural area of Xambrê, Paraná, Brazil (S2376'38.66" e W5365'62.96"), identified by PhD Lívia Godinho Temponi and deposited - number 2335 - at the Herbarium of the State University of Western Paraná. Meteorological data regarding the temperature, rainfall, and humidity (January 2016 to December 2016) were obtained from the State Agriculture and Supply Department-SEAB.

\section{Essential oil extraction}

The fresh leaves ( $250 \mathrm{~g}$ ) of $\mathrm{G}$. gardneriana were fragmented and submitted to the hydrodistillation in a Clevenger apparatus for $3 \mathrm{~h}$. At the end of each distillation, the EO was removed from the apparatus, transferred to amber vials to calculate the yield (\%) per total leaf biomass and was kept at the temperature of $-20^{\circ} \mathrm{C}$.

\section{Gas chromatography-mass spectrometry analysis}

The identification of chemical constituents of EO of "bacupari" leaves was carried out using a gas chromatograph (Agilent 7890 B) coupled to a mass spectrometer (Agilent $5977 \mathrm{~A}$ ) equipped with a HP-5MS UI Agilent column $(30 \mathrm{~m} \times 0.250 \mathrm{~mm} \times 0.25 \mu \mathrm{m})$ with the following conditions: injector temperature of $250^{\circ} \mathrm{C}$, injection volume of $2 \mu \mathrm{L}$ at a ratio of 1:10 (split mode) and carrier gas (helium) with flow of $1 \mathrm{~mL} \mathrm{~min}^{-1}$. The initial column temperature was $60^{\circ} \mathrm{C}$ and the column was gradually heated at a rate of $2^{\circ} \mathrm{C} \mathrm{min}-1$ until $160^{\circ} \mathrm{C}$. Thereafter, the rate used was $10^{\circ} \mathrm{C} \mathrm{min}{ }^{-1}$ until the temperature of $240^{\circ} \mathrm{C}$ was reached. The temperatures of the transfer line, ion source, and quadrupole were $260^{\circ} \mathrm{C}, 230^{\circ} \mathrm{C}$ and $150^{\circ} \mathrm{C}$, respectively. The detection system was mass spectra in the range of 29 $250(\mathrm{~m} / \mathrm{z})$ through scan mode with a solvent delay time of 3 $\mathrm{min}$. The compounds were identifed based on comparison of their retention index (RI), obtained using various $n$-alkanes (C7-C30). In addiction, their electron ionization mass spectra were compared with the NIST 11.0 library spectra and those reported by Adams (2007).

\section{Statistical analysis}

Principal component analysis (PCA) was applied to examine the interrelationships between the selected chemical constituents of the essential oils collected monthly (January to December/2016), and hierarchical cluster analysis (HCA) was used to verify the similarity of samples of different months based on the distribution of the selected chemical constituents, using the software Statistica version 7.0 Trial (StatSoft. Tulsa. OK. EUA).

The Pearson's correlation analysis was used to verify the possible combination between the essential oil components selected along with climatic variables (temperature and rainfall).

\section{Acknowledgements}

The authors thank the financial support of Coordenação de Aperfeiçoamento de Pessoal de Nível Superior (CAPES).

\section{References}

Abu-Darwish MS, Alu'datt MH, Al-Tawaha AR, Ereifej K, Almajwal A, Odat N, Al Khateeb W (2012) Seasonal variation in essential oil yield and composition from Thymus vulgaris $\mathrm{L}$. during different growth stages in the south of Jordan. Nat Prod Res. 26:1310-1317.

Aboaba S, Akande K, Flamini G (2014) Chemical composition, toxicity and antibacterial activity of the essential oils of Garcinia mangostana from Nigeria. J Essent Oil Bear. PI. 17: 78-86.

Adams RP (2012) Identification of essential oil components by gas chromatography/mass spectrometry. 4 ed. Allured Publishing Corporation, Illinois, USA. 804 p. 
Alves LF, Bonfim FPG, Oliveira SG, Campos MG, Bolina CO, Marques MOM (2018) Biomass, content, yield and chemical composition of mint (Mentha $\times$ villosa Huds.) essential oil in response to withholding irrigation. Aust J Crop Sci. 12: 519-523.

Asinelli MEC, Souza MC, Mourão KSM (2011) Fruit ontogeny of Garcinia gardneriana (Planch. \& Triana) Zappi (Clusiaceae). Acta Bot Bras. 25: 43-52.

Botta B, Mac-Quhae M, Delle Monache G, Delle Monache F, De Mello JF (1984) Chemical investigation of the genus Rheedia. V: biflavonoids and xanthochymol. J Nat Prod. 47: 1053.

Cechinel Filho V, Da Silva KL, De Souza MM, Oliveira $A E$, Yunes RA, Guimarães CL, Verdi LG, Simionatto EL, Delle Monache F (2000) I3-naringenina-II8-4'-OMe-eriodictyol: a new potential analgesic agent isolated from Rheedia gardneriana leaves. Z Naturforsch. 55c: 820-823.

Chagonda LS, Chalchat J-C (2005) The essential oil of the fruit of Garcinia huillensis Welw. ex. Oliv. from Zimbabwe. Flavour Fragr J. 20: 313-315.

Cheng A-X, Lou Y-G, Mao Y-B, Lu S, Wang L-J, Chen X-Y (2007) Plant terpenoids: biosynthesis and ecological functions. J Integr Plant Biol. 49: 179-186.

Corrêa MP (1984) Dicionário das plantas úteis da Brasil e das exóticas cultivadas. v. 6 Ministério da Agricultura, Rio de Janeiro, IBDF. p. 318-337.

Delle Monache G, Delle Monache F, Waterman PG, Crichton EG, Alves de Lima R (1984) Miner xanthones from Rheedia gardneriana. Phytochemistry. 23: 1759-1759.

Faraji R, Bigdelo M, Rezaei K, Hooshidari F, Mirzaei $\mathrm{HH}$ (2016) Essential oil composition of Leutea kurdistanica (Mozaff.) at the vegetative and flowering stages. J Essent Oil Bear PI. 19: 223-228.

Fernandes SR, Ferreira HD, Sá S, Borges LL, Tresvenzo LMF, Ferri PH, Santos PA, Paula JR, Fiuza TS (2017) Chemical composition and seasonal variation of the volatile oils from Trembleya phlogiformis leaves. Rev Bras Farmacogn. 27: 419-425.

Gobbo-Neto L, Lopes NP (2007) Medicinal plants: factors of influence on the content of secondary metabolites. Quim Nova. 30: 374-381.

Guimarães CL, Otuki MF, Beirith A, Cabrini DA (2004) Uma revisão sobre o potencial terapêutico da Garcinia gardneriana - NA. Dynamis Revista Tecno-Científica. 12: 612.

Hazzoumi Z, Moustakime Y, Amrani Joutei K (2015) Variation in yield and composition of essential oil in Pelargonium sp during the vegetative stage. CEBS. 132-135.
Heinzmann BM, Spitzer V, Simões CMO (2017) Óleos voláteis. In: Simões CMO, Schenkel EP, Mello JCP, Mentz LA, Petrovick PR. Farmacognosia: do produto natural ao medicamento. Porto Alegre, Artmed. $486 \mathrm{p}$.

Lorenzi H (2002) Árvores brasileiras: manual de identificação e cultivo de plantas arbóreas do Brasil. 4 ed. v.1. Instituto Plantarum, Nova Odessa. 368 p.

Luzzi R, Guimarães $C L$, Verdi LG, Simionatto EL, Delle Monache F, Yunes RA, Floriani AEO, Cechinel Filho V (1997) Isolation of biflavonoids with analgesic activity from Rheedia gardneriana leaves. Phytomedicine. 4: 139-142.

Matasyoh JC, Kiplimo JJ, Karubiu NM, Hailstorks TP (2007) Chemical composition and antimicrobial activity of the essential oil of Satureja biflora (Lamiaceae). Bull Chem Soc Ethiop. 21: 249-254.

Morais LAS (2009) Influência dos fatores abióticos na composição química dos óleos essenciais. Hort Bras. 27:S4050-S4063.

Olayemi RF (2017) The role of monoterpenoids and sesqiterpenoids as defense chemicals in plants - a review. NRJCS 3: 1-15.

Santos MH, Nagem TJ, Oliveira TT, Braz-Filho R (1999) 7epiclusionone, the new tetraprenylated benzophenone and others chemical constituents from the fruits of Rheedia gardneriana. Quím Nova. 22: 654-660.

Silva EB, Soares MG, Mariane B, Vallim MA, Pascon RC, Sartorelli $P$, Lago JH (2013) The seasonal variation of the chemical composition of essential oils from Porcelia macrocarpa R.E. Fries (Annonaceae) and their antimicrobial activity. Molecules. 18: 13574-13587.

Tan W-N, Wong K-C, Khairuddean M, Eldeen IM, Asmawi $M Z$, Sulaiman B (2013) Volatile constituents of the fruit of Garcinia atroviridis and their antibacterial and antiinflammatory activities. Flavour Fragr J. 28: 2-9.

Tan WN, Lim JQ, Afiqah F, Nik Mohamed Kamal NNS, Abdul Aziz FA, Tong WY, Leong CR, Lim JW (2018) Chemical composition and cytotoxic activity of Garcinia atroviridis Griff. ex T. Anders. essential oils in combination with tamoxifen. Nat Prod Res. 32: 854-858.

Ulubelen A, Topcu G, Eriş C, Sönmez U, Kartal M, Kurucu S, Bozok-Johansson C (1994) Terpenoids from Salvia sclarea. Phytochemistry. 36: 971-974.

Zhou W, Kügler A, McGale E, Haverkamp A, Knaden M, Guo $H$, Beran F, Yon $F$, Li R, Lackus N, Köllner TG, Bing J, Schuman MC, Hansson BS, Kessler D, Baldwin IT, Xu S (2017) Tissue-specific emission of $(E)$ - $\alpha$-bergamotene helps resolve the dilemma when pollinators are also herbivores. Curr Biol. 27: 1336-1341. 